\title{
Experimental performance of homothetic mapping for wide field interferometric imaging
}

\author{
Casper van der Avoort ${ }^{a, c}$, Hedser van $\mathrm{Brug}^{b}, \mathrm{Jan}$ Willem den $\operatorname{Herder}^{c}$, Luigi d'Arcio ${ }^{d}$, \\ Rudolf Le Poole ${ }^{e}$ and Joseph Braat ${ }^{a}$ \\ ${ }^{a}$ Delft University of Technology, Lorentzweg 1, 2628 CJ Delft, The Netherlands; \\ ${ }^{b}$ TNO-TPD, Stieltjesweg 1, 2628 CK Delft, The Netherlands; \\ ${ }^{c}$ Space Research Organisation of the Netherlands, Sorbonnelaan 2, \\ 3584 CA Utrecht, The Netherlands; \\ ${ }^{d}$ ESA-ESTEC, Keplerlaan 1, 2200 AG Noordwijk, The Netherlands; \\ ${ }^{e}$ Leiden Observatory, Niels Bohrweg 2, 2333 CA Leiden, The Netherlands
}

\begin{abstract}
Homothetic mapping is an aperture synthesis technique that allows interferometric imaging over a wide fieldof-view. A laboratory experiment was set up to demonstrate the feasibility of this technique. Here, we present the first static experiments on homothetic mapping that have been done on the Delft Testbed for Interferometry (DTI). Before a changeable telescope configuration is provided, we first took a fixed telescope configuration and tested the algorithms for their ability to provide an exit pupil configuration before beam combination, that was an exact copy of this telescope configuration. By doing so, we created a homothetic imaging system. This is an imaging system that acts as a masked aperture monolithic telescope, but consists of (in our case) three telescopes of which the light follow their own optical trains.
\end{abstract}

Keywords: Interferometry, homothetic mapping, wide field imaging, aperture synthesis

\section{INTRODUCTION}

Astronomical interferometry is a measurement technique that allows for measurements with very high angular resolution. When applied to imaging, the major drawback is the field-of-view of such an interferometer. Fortunately, solutions have been provided for this drawback. A cophased array of telescopes can be seen as physical apertures in a large synthetic aperture. When the light collected by all telescopes is properly combined, an image can be constructed with a resolution related to the diffraction limit of the synthetic aperture, see Fig. 1 . The physical apertures can be seen as holes in a mask on top of a general imaging system. If one can construct a system that lets the beams of light follow a path that they would also follow when they would really be part of that general imaging system, a correct image in the image plane will be constructed, with a very wide field-of-view. Making different beams of light act together as parts of a masked imaging system is called homothetic mapping.

With homothetic mapping, a stellar interferometer has theoretically a field-of-view that is basically only limited by the size of the detector. For imaging, but also for astrometry where more than one sources are to be measured at once, this feature is necessary. A telescope that demonstrates this principle is the Large Binocular Telescope $^{2}$ (LBT), be it that there are only two apertures present there and their baseline is fixed. The Delft Testbed for Interferometry (DTI, see Fig. 3) was constructed as a scaled copy of the VLTI, to demonstrate homothetic mapping with three baselines, see Van Brug et al.. ${ }^{1}$

We will now first provide more detail on homothetic mapping and the difficulties with it in Sec. 2. Then, the multi-baseline fringe measuring algorithm will be discussed in Sec. 3. In Sec. 4, the actuation of the exit pupil placement is clarified. Finally, our experimental results are shown in Sec. 5, demonstrating how the detection algorithm and pupil actuation work together to reach a homothetic configuration. We will conclude in Sec. 6 .

Further author information: (Send correspondence to Casper van der Avoort)

E-mail: c.vanderavoort@tnw.tudelft.nl, Telephone: +31 152789407 


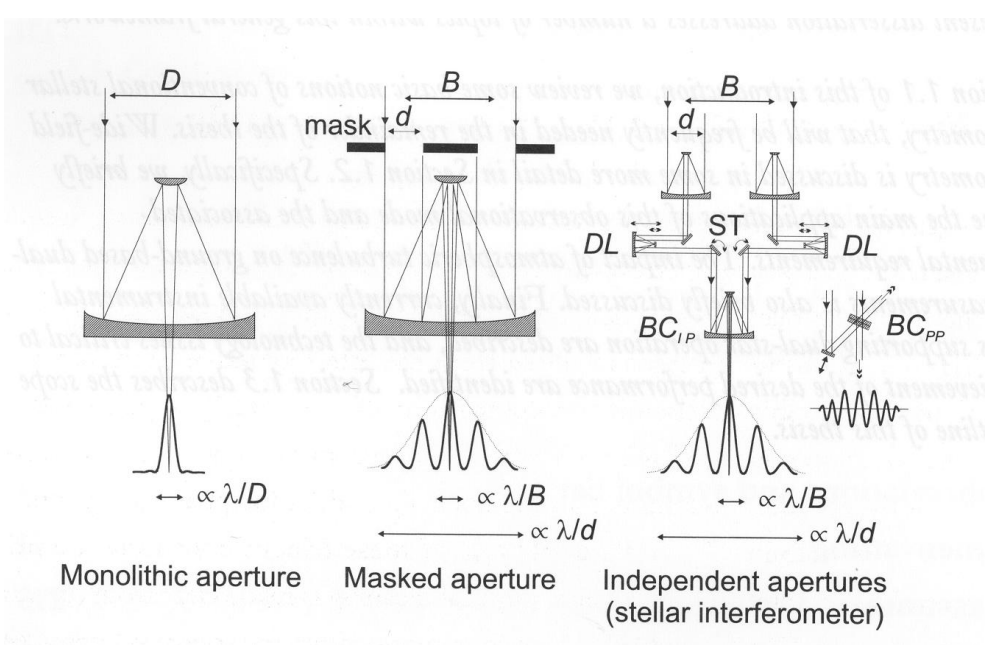

Figure 1. Imaging with a monolothic aperture and a masked aperture (left and central). On the right: conceptual equivalence of a synthetic aperture telescope with the masked aperture case. The indications $B C_{I P}$ and $B C_{P P}$ denote an Image-Plane Beam Combiner and a Pupil-Plane Beam Combiner. The latter is most common, but this work describes experiments on an Image-Plane interferometer.

\section{HOMOTHETIC MAPPING}

\subsection{Principles}

In order to image with a synthetic aperture $\left(\right.$ see $\left.\mathrm{Meinel}^{3}\right)$, the homotheticity conditions should be met. These can be described as: making sure that from input to exit pupils the system behaves as a regular imaging system. Otherwise, violation of these 'golden rules' ${ }^{4}$ would disqualify the system as performing proper imaging, and would lead to either a reduced field-of-view or poor and irreliable measurements.

For a certain magnification factor $\mathrm{M}$, the wavefront of an off-axis point source produces beams passing through the input (1) and exit (2) pupils. The diameter $D$, separation $B$ and direction $\alpha$ of these beams should match according to

$$
\begin{aligned}
B_{2} & =M \cdot B_{1} \\
\alpha_{1} & =M \cdot \alpha_{2} \\
\frac{B_{1}}{D_{1}} & =\frac{B_{2}}{D_{2}} .
\end{aligned}
$$

These relationships provide that all spatial information from the imaged object is preserved and that internal optical pathlength differences remain zero for all angles under which the sky is observed. Figure 2 shows what happens if the exit baseline length $B_{2}$ is not set properly. Controlling this length to have no field dependent OPD effects is a key issue.

DTI has a magnification of $M=1$. The next section will detail the tolerances needed for homothetic performance over the field-of-view of DTI. For an interferometer like VLTI, the magnification $M$ is rather large. There, for proper homothetic performance, the exit pupils need to be positioned with an accuracy of $1 / M$ times the accuracy required for the $M=1$ case $\left(\mathrm{d}^{\prime} \mathrm{Arcio}^{5}\right)$. However, the practical field-of-view of interest for such an interferometer is much smaller than that of the DTI.

When a smaller field-of-view is accepted, the absolute mapping condition can be relaxed. Experimentally, one can determine to what extent the mapping can be relaxed and hence at what tolerance the exit pupils need to be positioned. To arrive at this point, a detection system with dedicated algorithms is needed. From the formed image of on-axis and off-axis stars, the mapping quality should be derived and position feedback to the pupil locations is possible. 


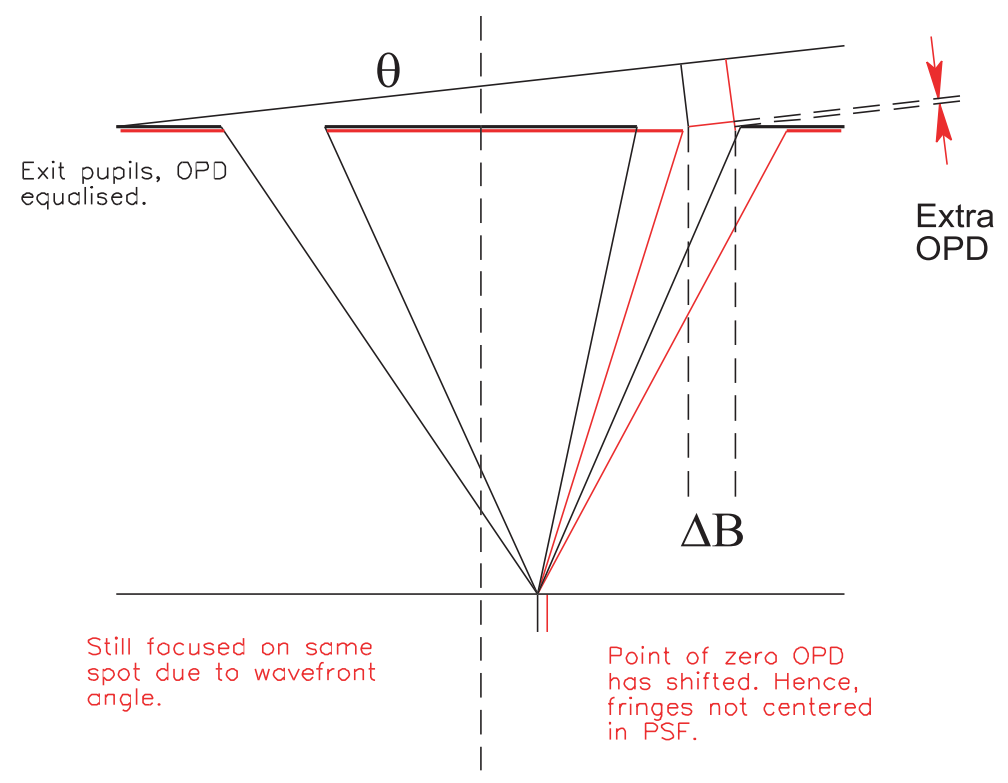

Figure 2. An improper placement of the exit pupils results in a measurable field dependent OPD effect. When the baseline extends (red compared to black) the star will be imaged on the same location, but there will be extra pathlength in one arm, which was not compensated for. This extra OPD for a mismapped exit baseline is proportional to the off-axis angle of the star.

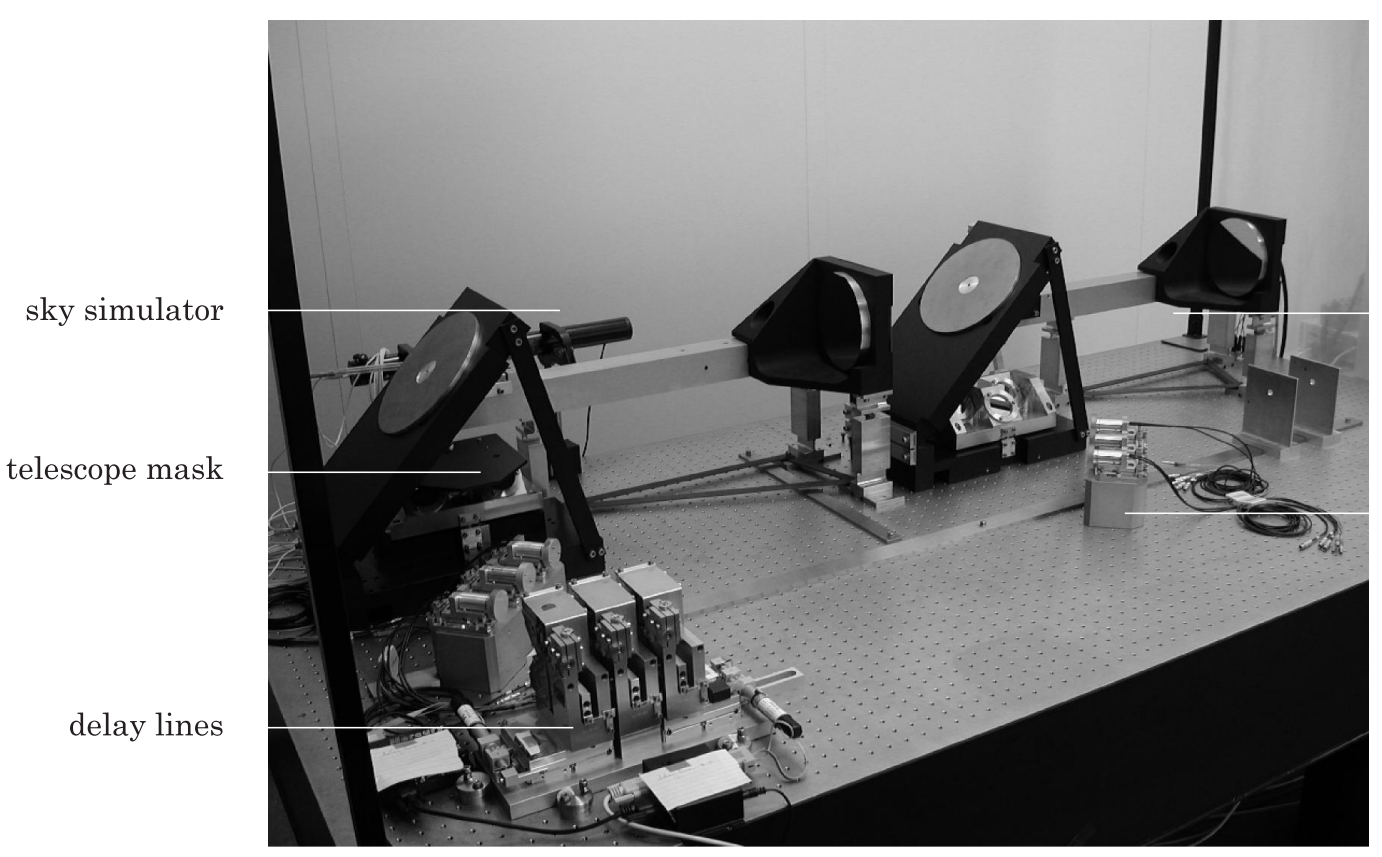

beam combiner

Figure 3. The experimental set-up for homothetic mapping, the Delft Testbed for Interferometry ${ }^{1}$ (DTI), as currently in the labs of TNO-TPD in Delft, the Netherlands. Systems for telescope movement and sky rotation are not shown in this picture. 
Table 1. Required tolerances for the testbed DTI to perform homothetic mapping upto a co-phased condition, defined as $1 / 20^{\text {th }}$ fringe movement. ${ }^{\text {A }}$ Field overlap: $10 \%$ of Airy diameter mismatch allowed

\begin{tabular}{|c|c|c|c|c|}
\hline $\begin{array}{l}\text { Tolerance criterion at field } \\
\alpha_{f} \text { is } 20 \mu \mathrm{rad}\end{array}$ & $\begin{array}{l}\text { Equivalent to the VLTI } \\
\text { Cophased Field-of-View } \\
\qquad \delta P<0.1 \mu \mathrm{m}\end{array}$ & culation in Beckers ${ }^{6}$ ) & Should match to & \\
\hline \multicolumn{5}{|l|}{$\alpha_{1}$ terms } \\
\hline Fractional baseline & $1.67 \cdot 10^{-4}$ & Exit baseline $\mathrm{S}^{*}$ & $10 \mathrm{~cm} \pm 16.7 \mu \mathrm{m}$ & (1) \\
\hline Fractional magnification & $1.67 \cdot 10^{-4}$ & Magnification M & $1 \pm 1.67 \cdot 10^{-4}$ & \\
\hline \multicolumn{5}{|l|}{$\alpha_{2}$ terms } \\
\hline \multicolumn{5}{|l|}{ Transverse exit pupil } \\
\hline placement $[\mathrm{mu}]$ & $5 \cdot 10^{4}$ & Pupil Piston & $0 \pm 25 \mathrm{~nm}$ & $(2)$ \\
\hline Fractional magnification & $5 \cdot 10^{-1}$ & (M less severe) & - & \\
\hline Image matching ${ }^{\mathbf{A}}$ & & $d \alpha=6.1 \mu r a d$ & & \\
\hline Fractional magnification & $8 \cdot 10^{-4}$ & Pupil tilt & $0 \pm 6.1 \mu \mathrm{rad}$ & $(3)$ \\
\hline Pupil rotation [degrees] & 0.045 & (M less severe) & - & \\
\hline \multicolumn{5}{|l|}{ Pupil rotation } \\
\hline$\phi_{\max }[$ degrees $]$ & .002 & Pupil rotation & $.23 \mathrm{deg}$ or $4 \mathrm{mrad}$ & $(4)$ \\
\hline$\delta \phi[$ degrees $]$ & .001 & & & \\
\hline$\delta P_{\text {tot }}[$ micron $]$ & $\begin{array}{l}0.22 \\
\text { Numbers in bold are }\end{array}$ & $\begin{array}{l}\text { Total fringe movement } \\
\text { ritical tolerances. }\end{array}$ & $1 / 4$ fringe & \\
\hline
\end{tabular}

\subsection{Tolerances}

We want to arrive at an interferometric cophased view of several Airy disks. For the VLTI, tolerances to achieve such cophasing were calculated by Beckers. ${ }^{6}$ The amount of pathlength differences and variations $(\delta P)$ across the field-of-view that can be allowed determine the tolerancing of the pupil configuration parameters. Beckers considers three cases for $\delta P$ :

1. A cophased field-of-view in which $\delta P<<1$ wavelength. In this case the white light fringe stays with a star image, to within a fraction of a fringe, when the star is moved across the field-of-view.

2. A highly coherent field-of-view in which $\delta P<$ a few wavelengths. In this case, all stars in the field-of-view have fringes, but the fringe-phase of a star depends on its location on the sky.

3. A poorly coherent field-of-view in which $\delta P<$ many wavelengths. In this case fringes may be visible on a star in the field-of-view, but might have moved off for another star.

Ad 1. The fraction by which the fringe is allowed to move depends on the application. For astrometry, more accurate fringe phase is needed than for imaging. For the DTI we chose the fraction to be $1 / 20$.

Ad 2. When the DTI was constructed and fringes were found using the coarse delay lines, this situation was already achieved. The optical alignment and mechanical construction lead to fringes on all stars in the field-of-view, be it clearly not centered on each star.

Beckers presented his findings in a table, where all sources of unwanted, field dependent OPD $(\delta P)$ are summed. Here we show this table with values for the case of DTI, in table 1 . The indications in bold refer to critical tolerances. The bold indications occur at different positions for the columns for VLTI and DTI, which relates to the hardware we used. 


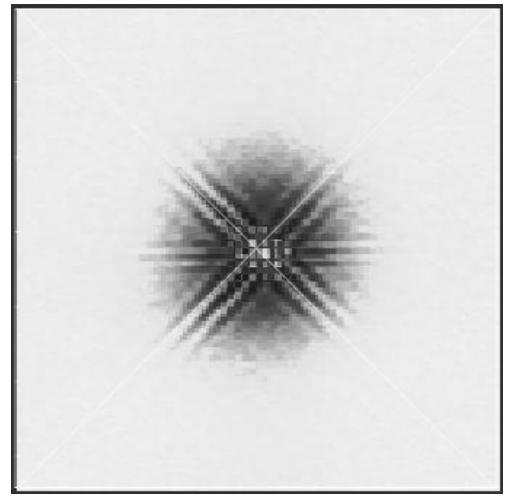

(a) Sub-image of a single star

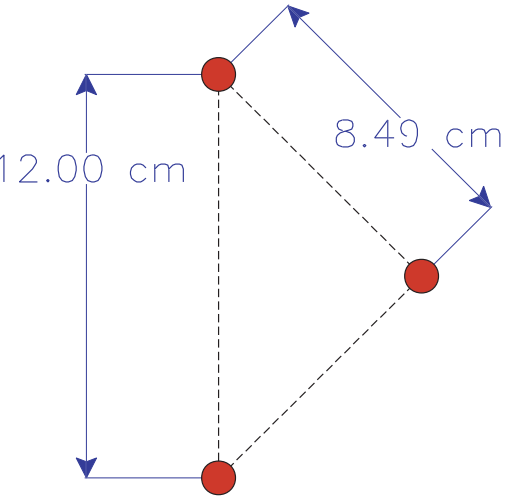

(b) Current telescope configuration

Figure 4. Typical image of a point source, imaged through the DTI system: (a) an Airy envelope and fringe packets across it, perpendicular to the three baseline directions (b).

Matching the baseline remains critical, but our $3 \mu \mathrm{rad}$ tilting mirrors with a separation of 1 meter give a high enough accuracy, even for the shorter baselines. The transverse exit pupil placement is not critical for DTI: both input and exit pupils do not have freedom to move out-of-plane and pathlengths can be controlled easily to be differing less than $25 \mathrm{~nm}$. Pupil rotation as mentioned for VLTI is also not critical because the symmetric lay-out of all elements prevents pupil rotation to occur in the amount as specified for DTI. However, another source for image mismatching is pupil tilt. Since our exit pupil placement system consists of tilting mirrors, it is necessary to monitor the occurrence of unwanted tilts. Both tilting mirror have an accuracy of $3 \mu \mathrm{rad}$ and the combined tilting of both (for pupil translation) is calibrated with a direct feedback on tilt, as described in Sec. 4 .

\section{DERIVING FRINGE PHASES}

The algorithm for deriving the fringe phases works on a two dimensional image from the CCD camera. It is based on processing the image in Fourier space. For clarity, the procedures will be explained by a one dimensional example. This linear example can be seen as an intersection through a two dimensional star image in the direction of any of the baselines.

\subsection{Obtaining a centered star-image}

The CCD provides an image of the sky, currently containing three stars, all resembling Fig. 4a. The coarse locations of these stars are easily picked and a small sub-image can be cut out around this coarsely picked location. By Fourier transforming such a sub-image, we can look at the spatial frequency contents of the subimage.

A star with fringes on it (see Fig. 5) can be seen as a superposition of information in two different spatial frequency regimes. The star envelope is relatively large with respect to the size of the sub-image and is represented in low spatial frequencies. The fringe pattern consists of narrow lines and is hence represented in higher spatial frequencies. The Fourier transformation of the data is given in Fig. 6. The amplitude part clearly shows the presence of the envelope in the low spatial frequencies (in the center) and the fringes at some higher frequencies (on two sides of the center).

In the definition of the Fourier transform, shifting a function yields an addition of a phase contribution linear with spatial frequency and proportional to the shift. Substitution of $x-x_{0}=u$ in the following expression identifies this phase slope addition:

$$
\int_{-\infty}^{\infty} f\left(x-x_{0}\right) e^{-2 \pi i k x} d x=\int_{-\infty}^{\infty} f\left(x-x_{0}\right) e^{-2 \pi i\left(x-x_{0}\right) k} e^{-2 \pi i\left(k x_{0}\right)} d\left(x-x_{0}\right)
$$




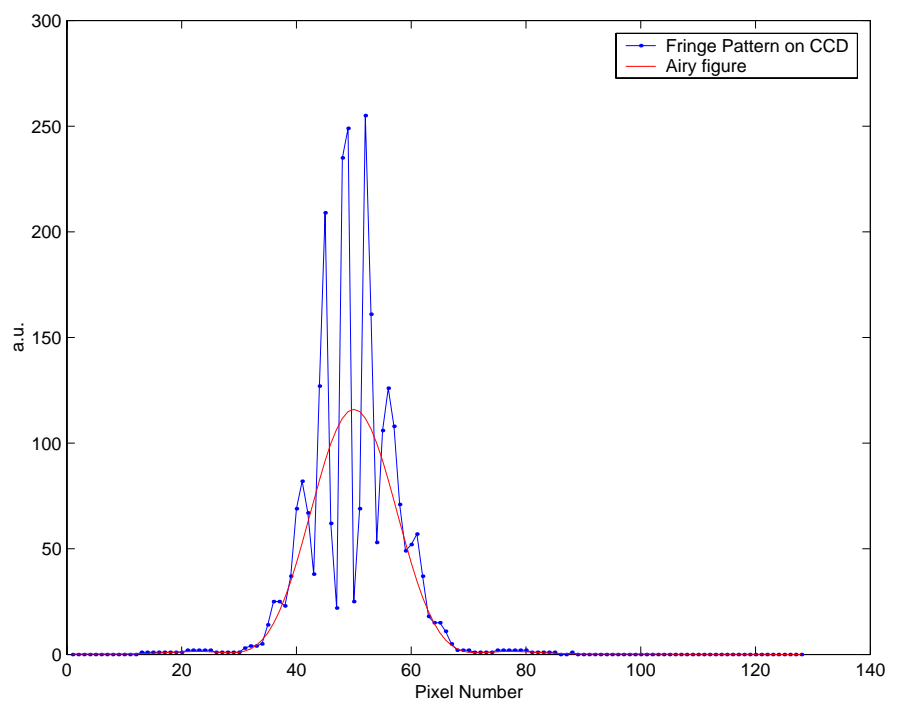

Figure 5. This dataset represents an Airy envelope with a packet of interference fringes in it. The envelope is not centered in the dataset (representing an unknown exact location of the star) and the fringe packet is not centered in the envelope, representing a non-zero OPD.

$$
=e^{-2 \pi i\left(k x_{0}\right)} \int_{-\infty}^{\infty} f\left(x-x_{0}\right) e^{-2 \pi i\left(x-x_{0}\right) k} d\left(x-x_{0}\right) .
$$

The use of this shifting theorem is the basis for both envelope position determination as well as fringe phase estimation. The Fourier amplitudes identify the regions of interest and the Fourier phases at those positions provide the amount of shift.

If the trend of the central lobe's phase function is measured and the total phase function (after unwrapping) is detrended according to this trend, the 'shift' of the total function is made undone. Moreover, the steepness of the trend is a measure for how far the envelope was out of the center. Together with the coarse estimated and cut-out location, this gives an accurate sub-pixel resolution position of the star.

The algorithm breaks down when the steepness is that high, that phase unwrapping in the central region is necessary. In practise, this occurs when the envelope is close to falling outside the sub-image. So when taking 128 pixels squared for a sub-image and having an Airy envelope of approximately 50 pixels, the coarse position estimation should be accurate up to 78 pixels, which is easily achievable.

\subsection{Obtaining the fringe packet position}

After the detrending of the total set, the remaining phase-trends in the sidelobes provide likewise the information of how far the fringe packet is off-center. Again, the shift property of the Fourier transform is applied, this time to the function representing the fringe packet. Figure 7 shows what happens in the regions of interest by thresholding the graphs of Fig. 6.

Whereas the total function was centered by fitting a line through the central phases, the retrieval of the fringe phase (or packet position) is a result of a fit through the periferic phases. But both fitting functions are a straight line through the origin. For steep lines (a packet far away from the center), phase unwrapping is necessary, starting from the origin. The algorithm breaks down at steepnesses that make the unwrapping break down. This will be when the packet is nearly off the envelope, so when the system is getting close to not be coherenced. The resolution and accuracy of this principle can get very high by taking more than one measurement and this allows cophasing up to the level that fringes are being detected at $1 / 20^{\text {th }}$ of a fringe-distance off center. 

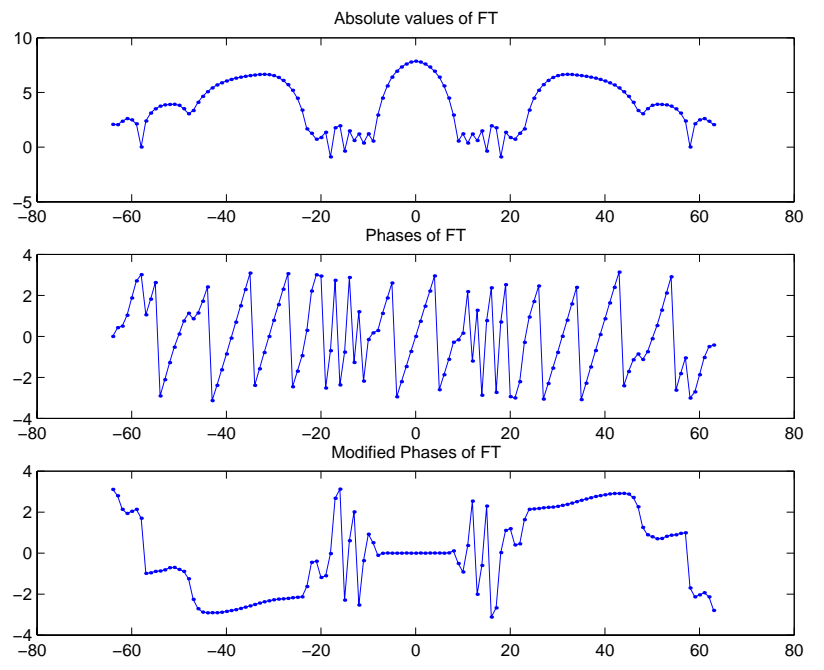

Figure 6. The representation of the dataset in Fig. 5 in Fourier amplitude and phase. Top: the amplitude part on a logarithmic scale. Middle: the phase information, wrapped between minus and plus $\pi$. Bottom: the processed phase information.
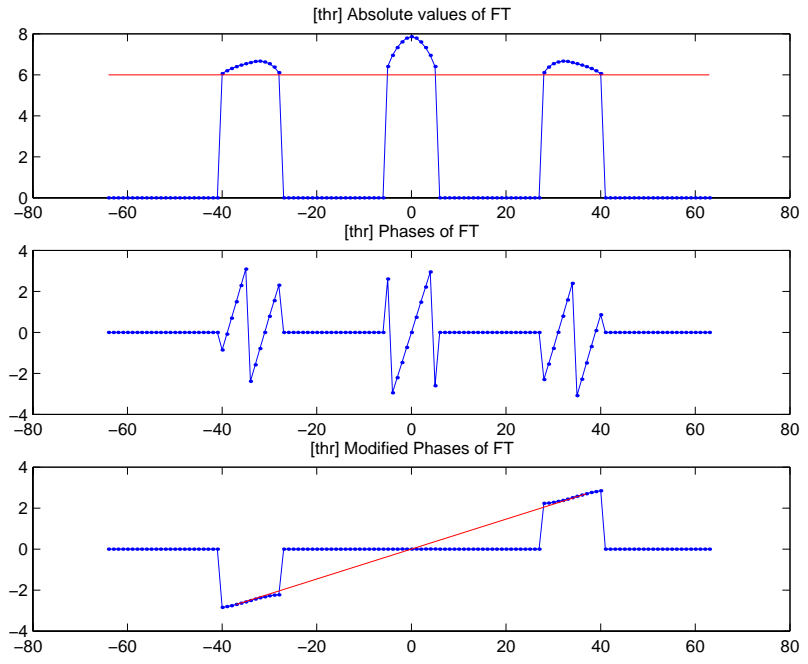

Figure 7. Thresholding the Fourier amplitude data clarifies the twofold trend retrieval. Top: the amplitude graph identifies a central lobe (containing the presence of the envelope) and two periferic lobes (for the presence of the fringe packet). Middle: the phase trend (present everywhere, but measured in the central lobe region) that is to be removed. Bottom: fitting a line through the phase information of the packet-lobes yields a measure for the packet position.

\section{MEASUREMENT AND ACTUATION}

As mentioned, wrong baseline lengths in the exit pupil configuration show up as field dependent OPD effects. The location of the optical axis is unknown and might even be projected outside the CCD. Hence, the origin of the angular coordinate (in the direction of each baseline) is hence unknown. The measured OPDs on all stars are a sum of true OPD between to beams, plus the field dependent OPD effect for which the origin of angular coordinates needs to be known. This requires that a baseline has to be set to at least two lengths, for which then the fringe positions on the stars are measured, before distinction can be made between true OPD and baselineerror-OPD. The location of the optical axis can be found by extrapolation of the obtained data. Additionally, since exit pupil displacement in the DTI is performed by a periscope system, at least four measurements on at least two different stars are needed. The following experiment will clarify this.

\subsection{Periscope}

The exit pupils are placed with a so-called periscope system of mirrors, as depicted in Fig. 8. By controlling the orientation of the two mirrors, one can influence the light coming out of it in three manners. The wavefront direction can be changed (by tilting either of the mirrors), the optical pathlength through this subsystem can be changed (by pistoning either of both mirrors) and the location of the virtual exit pupil can be changed by tilting both mirrors over exactly the same angle.

These 6 degrees of freedom, namely two orientations of the outcoming beam, two positions of the outcoming beam and two pistons of the mirrors, have to be controlled by 6 mounted actuators. A linearised system is valid for the small angles we envisage, so an easily solvable system can be constructed.

The six degrees of freedom are not all directly measureable. However, very good estimates can be made where necessary, as will be explained. Most important is the strict control of the direction of the light exiting the periscope. If any piezo movement results in a tip or tilt, the field overlap with the other beams is lost. This most important degree of freedom, tilt of the exit beam, is directly measureable on the camera, since a tilt will result in displacement of the diffraction spots in one beam. 


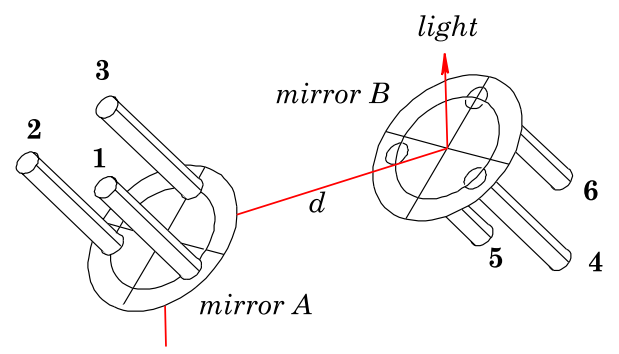

Figure 8. Sketch showing the periscope system for controlling the location and pointing of the exit pupils. The two mirrors A and B are actuated by six piezo electric actuators, mounted on the backside of the mirrors. Such a periscope is in place for each moving telescope, in our case of DTI this is two periscopes. The third beam is relayed by fixed mirrors.

The to be calibrated $6 \times 6$ control system will then look like

$$
\begin{aligned}
A \cdot p & =b \\
{\left[\begin{array}{ccc}
a_{1} & \cdots & a_{6} \\
& \ddots & \\
a_{31} & \cdots & a_{36}
\end{array}\right]\left(\begin{array}{c}
p_{1} \\
\vdots \\
p_{6}
\end{array}\right) } & =\left(\begin{array}{c}
\text { Tilt } \\
\text { Tip } \\
T_{x} \\
T_{y} \\
\text { Piston }_{A} \\
\text { Piston }_{B}
\end{array}\right)
\end{aligned}
$$

where $p_{i}$ is the length of piezo element $i$, ranging from 0 to $30 \mu \mathrm{m}$ and $a_{j}$ are the 36 unknowns. If we would now perform 36 measurements, where all $p_{i}$ take on random values and the measurement vector could be obtained, the unknowns could be deduced. However, as mentioned, the observables are partly only indirectly observable.

This problem is solved as follows. Firstly, the piston movement per mirror is taken to be the average of the lengths of the three piezos attached to it. Secondly, we abandon the idea of putting random values on the piezos. For all measurements, all piezos are first set to a central stretch of $15 \mu \mathrm{m}$. Then only one piezo is set to another length, making sure that the resulting tilt is not placing the spot off the camera. In this way, we know which mirror is moving, hence we can account for that in constructing the measurement vector:

$$
b_{123}=\left(\begin{array}{c}
\Delta X \\
\Delta Y \\
\Delta X \cdot d \\
\Delta Y \cdot d \\
\frac{1}{3}\left(p_{1}+p_{2}+p_{3}\right) \\
15
\end{array}\right), \quad b_{456}=\left(\begin{array}{c}
\Delta X \\
\Delta Y \\
0 \\
0 \\
15 \\
\frac{1}{3}\left(p_{4}+p_{5}+p_{6}\right)
\end{array}\right)
$$

In these expressions $\Delta X$ and $\Delta Y$ are the shifts of one focused spot on the camera. A tilt is thus expressed as a lateral shift, measured in units of pixels. Tilting mirror A results not only in a measurable tilt of the beam, but also in a displacement of the exit location, depending on mirror separation distance $d$.

\subsection{Measurement of exit pupils}

To reach homothetic conditions, the tilt mirros need to locate and point each beam (exit pupil) to the proper position and direction. It is required that this can be done automatically and for changing conditions. For correct homothetic mapping, we should observe stars over the total field with fringes on them according to the telescope and hence baseline pattern. Moreover, this fringe pattern should be exactly centered on the star, for each star.

The feedback signals required to tilt all piezo actuated mirrors are obtained from the image plane, by reading out the CCD camera. A number of stars $n$ can be picked and converted into $n$ sub images, originating from one 

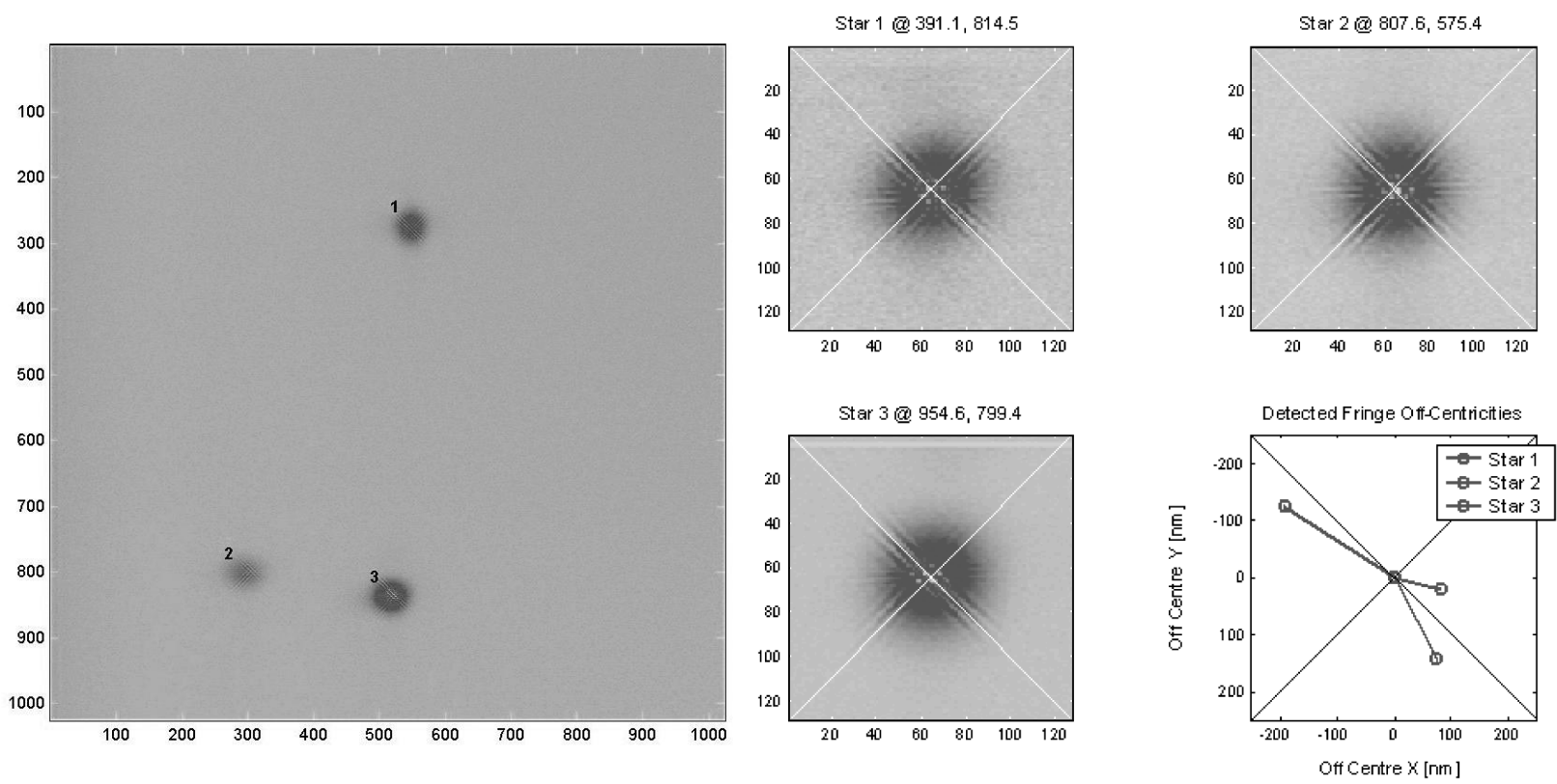

Figure 9. Stars can be picked from the CCD camera (left) and redirected into smaller sub-images (right) that are via FFT analysed to deliver (1.) the location of this star in the field and (2.) the fringe phases for all baselines. Units on all image axes are pixels.

large CCD recording. Then, each image is processed to deliver (1.) the location of this star in the field and (2.) the fringe phases for all baselines, as described in Sec. 3.

An OPD shows up as a fringe offset (2.) in the direction of that baseline, with the same amount for all stars. A misplacement of an exit pupil (non-homothetic situation) shows up as a fringe displacement (2.) per baseline direction that is proportional to the angular coordinate (1.) of that star perpendicular to that baseline, as shown in Fig. 2.

\section{VALIDATION EXPERIMENT}

The periscope movement induces a quadratic dependance of the optical pathlength with respect to the tilting angles of both mirrors or the parallel translation of the exit beam, where the latter is a linear and the desired dependence. This quadratic behaviour of the OPD of one moving beam with respect to one or more static beams was used as reference for the measurement and actuation system.

For the experiment, we measured the fringe phase in one baseline direction on two stars. Then, the length of this baseline was either stretched or shortened at the exit pupil plane, by making use of the periscope system. Note that from Fig. 2 we know that just for a baseline elongation $\triangle B$, the extra $\delta O P D$ for a star with an off-axis angle $\theta$ to that baseline is:

$$
\delta O P D=\Delta B \cdot \sin \theta,
$$

which is linear with respect to $\Delta B$ as well as star position on the sky, for small angles. Note also that a general OPD effect in the optical train results in a fringe shift that is equal for all stars. The OPD effect of our exit pupil placement method will therefore show up equally in all stars.

If both piezo calibration and fringe sensing algorithms are correctly implemented, the quadratic ODP dependence should be measurable and fitting to the expected curve, based solely on the separation $d$ of the tilting mirrors. The measured results and fitting process are depicted in Fig. 10. The residuals (Fig. 11) can be explained as results from slight misalignment in the beamcombiner. This is a parabolic-hyperbolic mirror pair, which is 


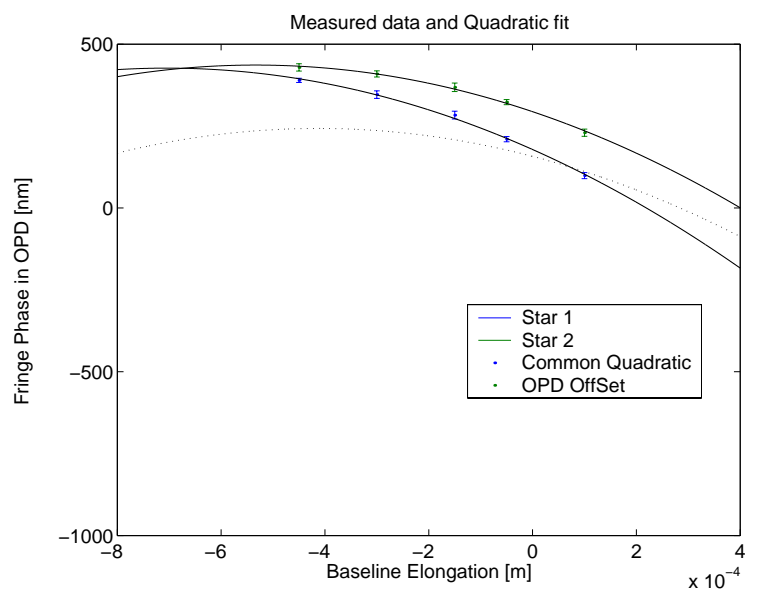

(a) Fit through measured data

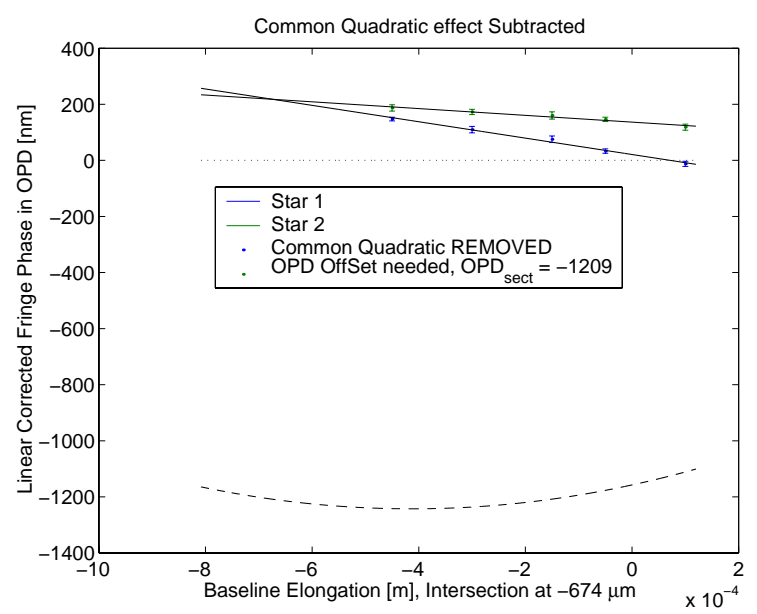

(b) Data corrected with parabola

Figure 10. The measured OPD effect of making a baseline shorter and longer is corrected for the parabolic OPD effect of the periscope. In figure (a) we see the measured OPD as a result of certain baseline lengths. The data are fit to a parabolic function (the same for both series) plus a linear part. In figure (b) this linear part clearly shows up, because the parabola is subtracted. Homothetic conditions are at the intersection of these lines: at this baseline length, the fringe phase in each star is equal and can be set to zero by adjusting the delay.

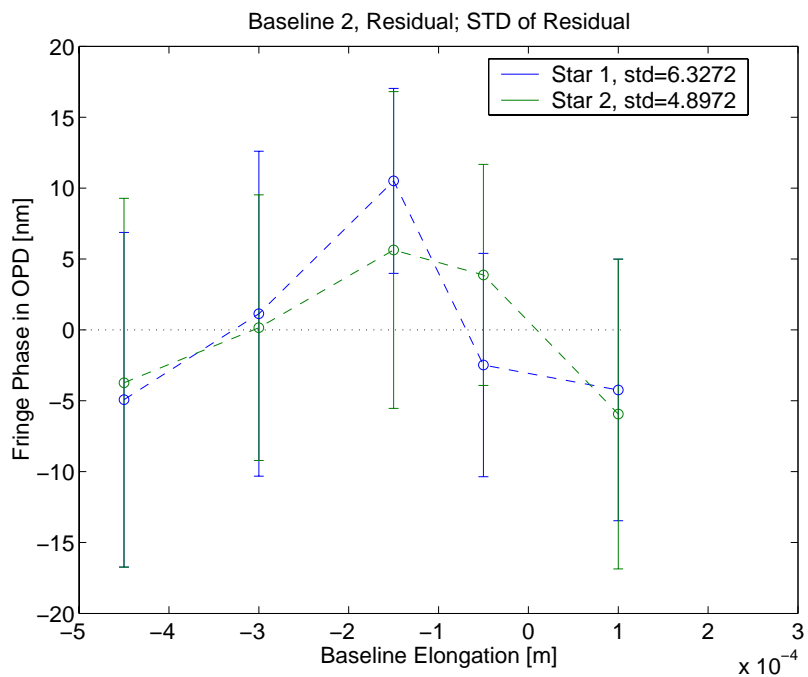

Figure 11. The deviation from the fit shown in figure 10b is depicted here. The RMS error of the fit is around $5 \mathrm{~nm}$, being $\lambda / 100$. The errorbars correspond to averaging 16 times per baseline setting. This figure shows that we can detect OPD effects in the nanometer regime, where micrometer disturbances are present, without placing the set-up in a conditioned room. 
known for its aberration sensitivity for small displacements of the mirrors. These aberrations will introduce small pupil-position dependent OPD effects that are not yet accounted for.

\section{CONCLUSIONS}

We have shown that the Delft Testbed Interferometer is accurately set up and that algorithms for detection and control are working. A simulated sky image through three telescopes can be obtained and all stars in the field show fringes. The stars can be acquired separately and fringe phase for all baselines can be measured with an accuracy that allows exit pupil positioning, so that the exit pupils before beam combination are an exact geometrical copy of the telescope pupils.

The testbed will finally perform dynamical remapping of a changing baseline configuration. The first statical experiments presented here already prove the feasibility of this when the hardware for arbitrary telescope configuration will be installed.

\section{ACKNOWLEDGMENTS}

The research described herein was performed mainly in the laboratories of TNO-TPD and Delft University of Technology. It was made possible by the Space Research Organisation of the Netherlands and was supported by the Knowledge centre for Aperture Synthesis, a collaboration of TNO-TPD, Delft University of Technology and Leiden Observatory.

\section{REFERENCES}

1. H. van Brug, T. van den Dool, W. Gielesen, P. Giesen, B. Oostdijck, and L. d'Arcio, "Delft testbed interferometer - layout design and research goals," Interferometry for Optical Astronomy II, SPIE 4838, pp. 425 - 429, August 2002.

2. J.R.P. Angel, J.M. Hill, P.A. Strittmatter, P. Salinari and G. Weigelt, "Interferometry with the large binocular telescope," Astronomical Interferometry, SPIE 3350, pp. 881-889, 1998.

3. A. Meinel, "Aperture synthesis using independent telescopes," Applied Optics 9, pp. 2501-2504, 1970.

4. W. Traub, "Combining beams from separated telescopes," Applied Optics 25, pp. 528-532, February 1986.

5. L.A. d'Arcio, Selected aspects of wide-field stellar interferometry. PhD thesis, Delft University of Technology, November 1999.

6. J.M. Beckers, "The VLTI, part III: Factors affecting wide field-of-view operation," Advanced Technology Optical Telescopes IV, SPIE 1236, pp. 379-389, 1990. 